\title{
PERFORMANCE OF PRECISE POINT POSITIONING WITH AMBIGUITY RESOLUTION FOR 1- TO 4-HOUR OBSERVATION PERIODS
}

\author{
J. Geng, X. Meng, F. N. Teferle, A. H. Dodson
}

Institute of Engineering Surveying and Space Geodesy, University of Nottingham, UK

\begin{abstract}
Recent progress in integer ambiguity resolution at a single station has made it possible to achieve high positioning accuracy in static precise point positioning (PPP) using a short period of observations. In this paper, 12 stations across Europe are used to conduct short-period (i.e. one, two, three and four hours) static PPP with ambiguity resolution from Day 245 to 251 in 2007. It is demonstrated that, when over three hours of observations are used, PPP can achieve a success rate of 100\% for ambiguity resolution, a $3 D$ positioning accuracy of about $1.0 \mathrm{~cm}$ and an occurrence of less than $1.0 \%$ for degraded solutions. Moreover, for the fixed solutions, increasing the observation period hardly improves the horizontal positioning accuracy while still improving the vertical one. Therefore, it is proposed that at least three hours of observations should be used in the ambiguity-fixed static PPP if a reliable millimetre positioning accuracy is required in the engineering applications.
\end{abstract}

KEYWORDS. Precise point positioning. Ambiguity resolution. Short observation period. GPS.

\section{INTRODUCTION}

During the past three decades, the development in the processing strategy for Global Positioning System (GPS) measurements has led to the products of highly accurate satellite orbits, satellite clocks, and Earth rotation parameters (ERP). The International GNSS Service (IGS) and its Analysis Centres routinely generate these products which are the basis for the development of precise point positioning (PPP) [28]. During the PPP data processing, these products are employed to implement absolute and accurate positioning at only one single station without any synchronous GPS observations from the base stations. Thus PPP has become the foremost choice in some areas, such as offshore and desert areas, where a nearby base station is unavailable and its establishment is difficult or not cost-effective.

It is well-known that PPP is capable of providing millimetre positioning accuracy in the static mode using 24 hours of observations [15]. Nevertheless, such daily observations are normally unavailable or impractical in most engineering applications where rapid static positioning is usually required [17]. In practice, at most a few hours of observing work are likely to be carried out in a field survey ([5], [7], [21]). Consequently, the static positioning accuracy within such a short observation period can hardly achieve millimetre level if PPP has to be applied to this field survey. For instance, [13] and [23] reported that hourly position estimates in PPP could only achieve sub-decimetre accuracy, and 4-hourly position estimates were at the level of centimetre accuracy. On the contrary, static relative positioning can provide centimetre accuracy using only 15 minutes of observations when double-difference ambiguity resolution is applied to a baseline of a few tens of kilometres [17]. Hence, it can be considered reasonable that integer ambiguity resolution in PPP is also able to significantly improve the positioning accuracy within a short observation period.

However, to date, integer ambiguity resolution in PPP is largely ignored due to the fact that the non-integer receiver- and satellite-dependent uncalibrated phase delays (UPD) [1], which are related to hardware [8], are absorbed by the real-valued 
ambiguity estimates, thus destroying the integer properties of the ambiguities ([8], [9]). Fortunately, recent studies have revealed that integer ambiguity resolution at a single station is possible if these UPD can be precisely determined in advance with a network of base stations ([3], [9], [16]). [9] suggested that the fractional parts of the UPD between satellites could be computed by averaging the fractional parts of all involved real-valued ambiguity estimates. As a comparison, [3] and [16] used the satellite clock estimates to assimilate the UPD by constraining the ambiguities at the base stations to integer values. [4] showed that $90 \%$ of hourly horizontal position estimates were at the level of 2-cm accuracy after integer ambiguity resolution. [11] and [12] showed that through ambiguity resolution the hourly positioning accuracy could be improved from $3.8 \mathrm{~cm}, 1.5 \mathrm{~cm}$ and $2.8 \mathrm{~cm}$ in the float solutions to $0.5 \mathrm{~cm}, 0.5 \mathrm{~cm}$ and $1.4 \mathrm{~cm}$ in the fixed solutions for the East, North and Up components, respectively.

This paper aims at comparing the performance of ambiguity-fixed static PPP with different short observation periods (i.e. one, two, three and four hours), including the efficiency and reliability of ambiguity resolution, the improvement of positioning accuracy and the occurrence of degraded solutions. In the following sections, the method adopted in this study for PPP ambiguity resolution is firstly introduced. Then the efficiency and reliability of ambiguity resolution, the positioning accuracy and the degraded solutions in short-period static PPP are presented and discussed.

\section{DETERMINATION OF UNCALIBRATED PHASE DELAYS}

In general, the one-way GPS observation equation for carrier phase data in the unit of length from receiver $k$ to satellite $i$ is written as [9]

$$
L_{m k}^{i}=\rho_{k}^{i}-\frac{\kappa}{f_{m}^{2}}+\lambda_{m} b_{m k}^{i} .
$$

where $m$ denotes the frequency band with corresponding wavelength $\lambda_{m}$ and frequency $f_{m} ; \rho_{k}^{i}$ represents the non-dispersive delay, mainly including the geometric distance, the receiver and satellite clocks and the tropospheric delay; $\frac{\kappa}{f_{m}^{2}}$ denotes the first order ionospheric delay, and the higher order delays are ignored; $b_{m k}^{i}=n_{m k}^{i}+\phi_{m k}-\phi_{m}^{i}$ in which $n_{m k}^{i}$ is the integer ambiguity, $\phi_{m k}$ is the receiverdependent UPD and $\phi_{m}{ }^{i}$ is the satellite-dependent UPD, and thus $b_{m k}{ }^{i}$ is usually recognized as a real-valued carrier phase bias term that is constant during its corresponding observing session. For briefness, multipath effects and measurement errors are not written explicitly in Equation 1.

For dual frequency data, the ionosphere-free combination observable is usually used to eliminate the first order ionospheric delay. Its carrier phase bias term in the unit of cycles reads

$$
b_{c k}^{i}=\frac{f_{1}^{2}}{f_{1}^{2}-f_{2}^{2}} b_{1 k}^{i}-\frac{f_{1} f_{2}}{f_{1}^{2}-f_{2}^{2}} b_{2 k}^{i}
$$

which can be reformulated as

$$
b_{c k}^{i}=\frac{f_{1}}{f_{1}+f_{2}} b_{n k}^{i}+\frac{f_{1} f_{2}}{f_{1}^{2}-f_{2}^{2}} b_{w k}^{i}
$$

where $b_{n k}^{i}$ is the narrow-lane (NL) carrier phase bias and $b_{w k}^{i}$ is the wide-lane (WL) one. To remove the receiver-dependent UPD, between-satellite differences (BSD) are applied to the one-way ambiguity estimates at receiver $k$. Thus Equation 3 becomes 


$$
b_{c k}^{i, j}=\frac{f_{1}}{f_{1}+f_{2}}\left(n_{n k}^{i, j}-\phi_{n}^{i, j}\right)+\frac{f_{1} f_{2}}{f_{1}^{2}-f_{2}^{2}}\left(n_{w k}^{i, j}-\phi_{w}^{i, j}\right)
$$

where $\phi_{n}^{i, j}$ denotes the BSD NL UPD and $\phi_{w}^{i, j}$ denotes the BSD WL UPD. In the following, 'NL UPD' denotes 'BSD NL UPD' and 'WL UPD' denotes 'BSD WL UPD' for briefness.

In this paper, the Melbourne-Wübbena combination observable ([19], [27]) is used to determine the WL UPD. That is

$$
\phi_{w}^{i, j}=\left\langle\left[b_{w k}^{i, j}\right]-b_{w k}^{i, j}\right\rangle
$$

where $\langle\cdot\rangle$ denotes averaging over all involved stations; [.] denotes the rounding operation. It has been confirmed that daily mean WL UPD are quite stable over a rather long period (e.g. at least several days to even several months) ([8], [9], [16]).

Once $\phi_{w}^{i, j}$ is determined, $n_{w k}^{i, j}$ can then be fixed to an integer. Thus Equation 4 becomes

$$
n_{n k}^{i, j}-\phi_{n}^{i, j}-\frac{f_{2}}{f_{1}-f_{2}} \phi_{w}^{i, j}=\frac{f_{1}+f_{2}}{f_{1}} b_{c k}^{i, j}-\frac{f_{2}}{f_{1}-f_{2}} n_{w k}^{i, j}
$$

The left side of this equation can be redefined as the difference between an integer part $n_{(n w) k}^{i, j}$ and a fractional part $\phi_{(n w)}^{i, j}$. In this paper $\phi_{(n w)}^{i, j}$ is still called NL UPD which is estimated using

$$
\phi_{(n w)}^{i, j}=\left\langle\left[\frac{f_{1}+f_{2}}{f_{1}} b_{c k}^{i, j}-\frac{f_{2}}{f_{1}-f_{2}} n_{w k}^{i, j}\right]-\frac{f_{1}+f_{2}}{f_{1}} b_{c k}^{i, j}+\frac{f_{2}}{f_{1}-f_{2}} n_{w k}^{i, j}\right\rangle
$$

Unlike WL UPD, NL UPD are normally estimated within a short observation period, such as 15 minutes, to obtain high estimation precisions [9]. However, [12] found that the NL UPD are rather stable during each full pass of a satellite pair over a regional network, and thus one NL UPD is estimated within one such pass. This strategy is also adopted in this study.

\section{AMBIGUITY RESOLUTION IN PPP}

After the WL and NL UPD are determined precisely, WL and NL ambiguity resolution at a single station can be implemented sequentially. The integer property of a WL ambiguity is recovered using

$$
n_{w k}^{i, j}=b_{w k}^{i, j}+\phi_{w}^{i, j}
$$

The WL ambiguity resolution follows the sequential bias fixing strategy [6]. If $n_{w k}^{i, j}$ is fixed to an integer successfully, then the integer property of its corresponding NL ambiguity is recovered by

$$
n_{(n w) k}^{i, j}=\frac{f_{1}+f_{2}}{f_{1}} b_{c k}^{i, j}-\frac{f_{2}}{f_{1}-f_{2}} n_{w k}^{i, j}+\phi_{(n w)}^{i, j}
$$

Due to the high correlation between the ambiguities in the short-period PPP data processing, a search strategy based on the LAMBDA (Least-squares AMBiguity Decorrelation Adjustment) method [24] is applied to conduct the NL ambiguity resolution.

For the ambiguity validation, two statistical tests are used, and the integer candidates are accepted only when both tests are passed in this study. One test is based on the compatibility between the unit variances of the fixed and float solutions ([14], [25]), which is 


$$
\frac{\sigma_{\text {fixed }}^{2}}{\sigma_{\text {float }}^{2}}<\frac{m}{r-t}\left(F_{m, r-t-m ; \alpha}-1\right)+1
$$

where $r$ is the number of observations, $m$ is the number of ambiguity parameters and $t$ is the number of the remaining parameters; $F_{m, r-t-m ; \alpha}$ denotes the F-distribution of a confidence level $\alpha$ with $m$ and $r-t-m$ degrees of freedom. Furthermore,

$$
\frac{\sigma_{\text {fixed }}^{2}}{\sigma_{\text {float }}^{2}}=\frac{r-t-m}{r-t} \cdot \frac{\Omega_{\text {float }}+R}{\Omega_{\text {float }}}
$$

where $\Omega_{\text {float }}$ is the quadratic form of the residuals in the float solution; $R=(\hat{\mathbf{n}}-\mathbf{n})^{T} \mathbf{Q}_{\hat{\mathbf{n}}}^{-1}(\hat{\mathbf{n}}-\hat{\mathbf{n}})$ in which $\hat{\mathbf{n}}$ denotes the real-valued ambiguity vector; $\mathbf{n}$ denotes the integer candidate vector and $\mathbf{Q}_{\hat{\mathbf{n}}}$ denotes the variance-covariance matrix of $\hat{\mathbf{n}}$ [14]. Actually, $R$ denotes the distance between $\hat{\mathbf{n}}$ and $\mathbf{n}$ under the metric of $\mathbf{Q}_{\hat{\mathbf{n}}}$, and thus a smaller $R$ indicates that the real-valued ambiguity estimates are more close to the optimum integer candidates. In other words, a smaller $\frac{\sigma_{\text {fixed }}^{2}}{\sigma_{\text {float }}^{2}}$ indicates a more reliable ambiguity resolution.

However, in this study this test often failed even when correct integer ambiguities were found, implying that the right side of Equation 10 might be too conservative. To solve this problem, [25] suggested using more observations, but this is not feasible for short-period static PPP. As a trade-off between maximizing the pass rate in the ambiguity validation and minimizing the number of the incorrectly fixed solutions, a critical value of 1.8 , which was empirically determined in terms of all $\frac{\sigma_{\text {fixed }}^{2}}{\sigma_{\text {float }}^{2}}$ estimates derived from all solutions in this study, was set instead of the right side of Equation 10.

On the other hand, we also used the well-known ratio test which is generally defined as the ratio of the second minimum quadratic form of the residuals to the minimum quadratic form of the residuals for the fixed solution. It is used to discriminate between the second optimum set of integer candidates and the optimum one. In this study, we used $\frac{R_{o p t 2}}{R_{o p t 1}}>c$ instead, where $R_{o p t 1}$ and $R_{o p t 2}$ correspond to the optimum and the second optimum integer candidates, respectively [14]. Due to the unknown statistical distribution of $\frac{R_{o p t 2}}{R_{o p t 1}}([\mathbf{2 5}],[\mathbf{2 6}])$, in this paper the critical criterion $c$ is set to 3 which is generally deemed as conservative in ambiguity validation [14]. The ratio value is usually considered as an index of denoting the reliability of ambiguity resolution, and a larger ratio value indicates a more reliable ambiguity resolution.

\section{DATA AND MODELS}

In this study, the PANDA (Positioning And Navigation Data Analyst) software [10], originally developed at Wuhan University in China, is utilized to test short-period static PPP with ambiguity resolution. It is a versatile tool for the scientific analysis of GPS positioning and navigation data, and currently serves as a fundamental platform for scientific studies in China and several international research centres.

Figure 1 shows a network of about 80 stations from the EUREF (European Reference Frame) Permanent Network (EPN) [2] of which daily observations covering 
Day 245 to 251 in 2007 were used to determine the WL and NL UPD. We also used the final products of the satellite orbits and clocks, the ERP and the differential code biases produced by CODE (Centre for Orbit Determination in Europe). Meanwhile, we applied the absolute antenna phase centres, the phase wind-up corrections and the station displacement conventions from the International Earth Rotation and Reference System Service [18]. The elevation cut-off angle for usable data was seven degrees. The estimated parameters included the positions, the receiver clocks, the zenith tropospheric delays, the horizontal troposphere gradients and the ambiguities. All stations that use cross-correlation receivers were excluded due to their relatively poor pseudo-range quality [9].

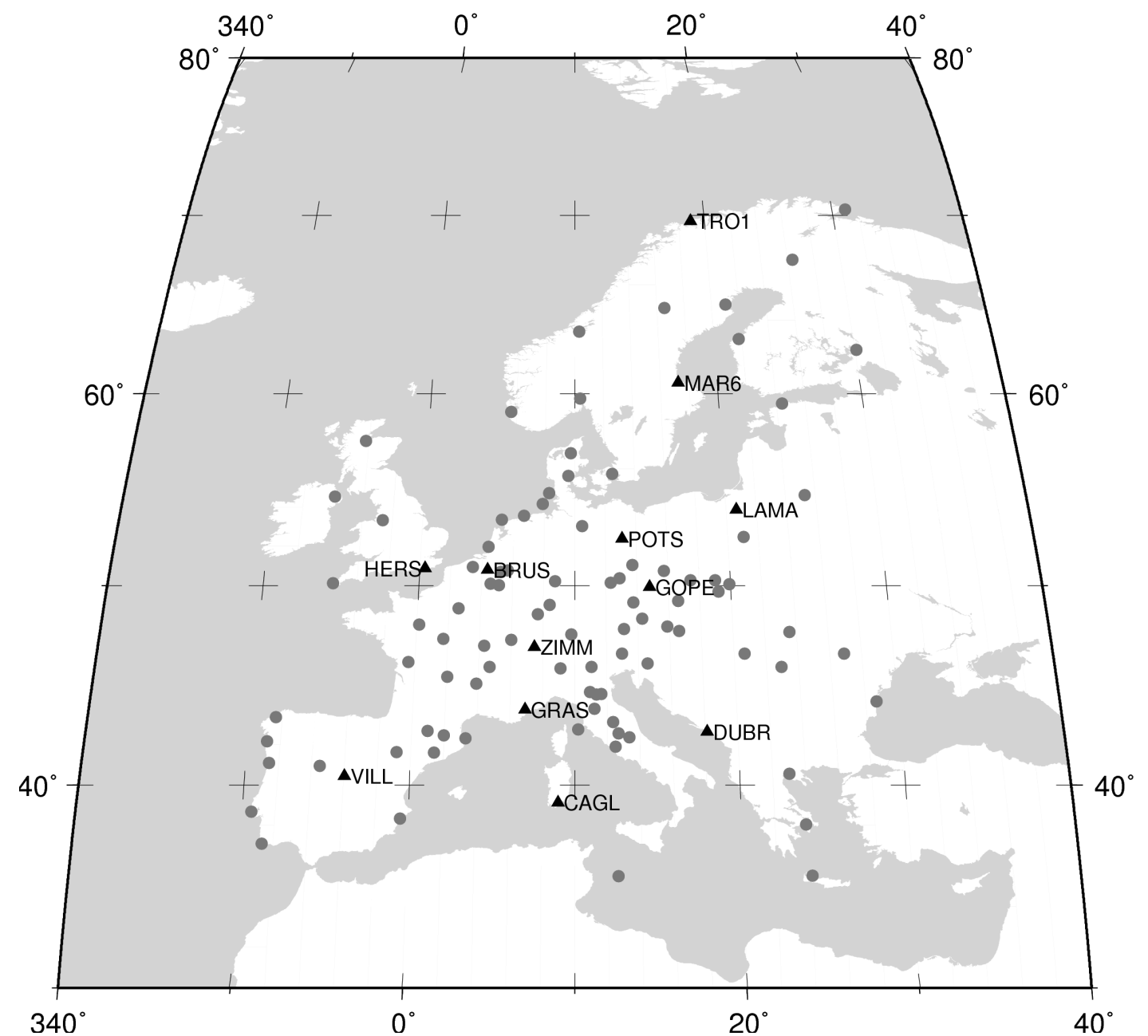

Fig. 1. Station distribution. The solid circles denote the EPN stations used for the determination of uncalibrated phase delays (UPD) whilst the solid triangles with names aside denote the IGS stations for testing the short-period static PPP with ambiguity resolution

In order to assess the performance of ambiguity resolution when utilizing these regional UPD estimates, we selected 12 stations from the IGS network (Figure 1) which were not used for the UPD determination to conduct short-period static PPP from Day 245 to 251 in 2007 . Hence, there were 168 hourly, 84 2-hourly, 56 3-hourly and 42 4-hourly solutions for each station if there were no large data gaps. We removed the solutions when the data gaps are longer than half of the required observation period, or when less than five satellites were available during more than half of the required observation period. The 12 stations were distributed evenly within the coverage of the EPN. The models adopted at these stations were the same as those for the EPN stations except for the horizontal troposphere gradients, which cannot be 
estimated precisely using only a few hours of observations. The estimated parameters and their a priori constraints are shown in Table 1.

Table 1. Parameters estimated in short-period static PPP

\begin{tabular}{ll}
\hline Parameters & Models \& a priori constraints \\
\hline Static position & 1 metre for each component \\
Receiver clock & White noise, 9000 metres \\
Zenith tropospheric delay & $\begin{array}{l}\text { Constant within each hour, } 20 \mathrm{~cm}, \text { Niell mapping } \\
\text { function }[\mathbf{2 0}]\end{array}$ \\
Ambiguity & 10000 cycles \\
\hline
\end{tabular}

In order to assess the accuracy of the position estimates, the daily estimates were used as the truth benchmarks instead of the official EUREF estimates to avoid potential biases between our solutions and the EUREF ones [22].

\section{RESULTS AND DisCUSSION}

In the following sections, the efficiency of ambiguity resolution, the reliability of ambiguity resolution, the positioning accuracy and the occurrence of degraded solutions are presented and discussed sequentially.

\section{Efficiency of ambiguity resolution}

Table 2 shows the number of all solutions, the number of the solutions with successful ambiguity resolution, the number of the solutions without any ambiguities fixed, the number of the solutions with incorrect ambiguity resolution and the number of the outlier solutions in PPP for different short observation periods at all test stations. In this study, successful ambiguity resolution denotes not only correct integer resolution, but also a 3D positioning accuracy of better than $10 \mathrm{~cm}$ in the float or fixed solution, or in both; incorrectly fixed ambiguities are identified using the integer ambiguity estimates derived from daily data processing; and outlier solutions denote those in which the ambiguities are fixed correctly, but the 3D positioning accuracies in both float and fixed solutions are worse than $10 \mathrm{~cm}$. This 10-centimetre threshold is chosen in terms of the normal 3D position accuracy derived in hourly PPP ([13], [23]).

In Table 2, when the observation period is increased from one hour to two, three and four hours, the percentage of the solutions with successful ambiguity resolution is increased from $97.9 \%$ to $99.6 \%, 100.0 \%$ and $100.0 \%$, respectively. The number of the failed solutions, shown in the last three columns of Table 2 , decreases to zero when the observation period is over three hours. Hence, it can be concluded that longer observation periods are conducive to higher success rates of ambiguity resolution in short-period static PPP, and a $100.0 \%$ success rate is achievable when over three hours of observations are used.

Table 2. Numbers of all solutions, solutions with successful ambiguity resolution, solutions without any ambiguities fixed, solutions with incorrect ambiguity resolution and outlier solutions for different short observation periods

\begin{tabular}{llllll}
\hline Observation period & Total & Successful & Unfixed & Incorrect & Outlier \\
\hline One hour & 1992 & 1951 & 8 & 17 & 16 \\
Two hours & 1001 & 997 & 0 & 2 & 2 \\
Three hours & 667 & 667 & 0 & 0 & 0 \\
Four hours & 501 & 501 & 0 & 0 & 0 \\
\hline
\end{tabular}




\section{Reliability of ambiguity resolution}

Figure 2 shows the mean values of the test statistics for ambiguity validation in all fixed solutions at all test stations when different short observation periods are used.

The black bars denote the $\frac{\sigma_{\text {fixed }}^{2}}{\sigma_{\text {float }}^{2}}$ test statistic whilst the grey bars denote the $\frac{R_{\text {opt } 2}}{R_{\text {opt } 1}}$ test statistic. With the hourly gradual increase of the observation periods from one to two, three and four hours, $\frac{\sigma_{\text {fixed }}^{2}}{\sigma_{\text {float }}^{2}}$ decreases from 1.19 to $1.15,1.13$ and 1.12 , respectively. In terms of Equation 11, this decreasing trend of $\frac{\sigma_{\text {fixed }}^{2}}{\sigma_{\text {float }}^{2}}$ suggests that the increase of the observation period contributes to more reliable ambiguity resolution in the shortperiod static PPP.

On the other hand, Figure 2 shows that all the $\frac{R_{o p t 2}}{R_{o p t 1}}$ test statistics are significantly larger than the chosen criterion of 3 for all different observation periods. These results demonstrate that ambiguity resolution at the test stations within a short observation period is rather reliable. Nevertheless, $\frac{R_{o p t 2}}{R_{o p t 1}}$ increases first, but then decreases when the observation period is over two hours. Apparently, it appears that ambiguity resolution with longer observation period is even less reliable than that with only two hours of observations, which is not reasonable. Actually, this issue can be explained in terms of the different degrees of freedom for the $\frac{R_{o p t 2}}{R_{o p t 1}}$ test statistics when different periods of observations are used. Therefore, the statistical distributions of these $\frac{R_{o p t 2}}{R_{o p t 1}}$ test statistics are totally different from each other, leading to no comparability between them.

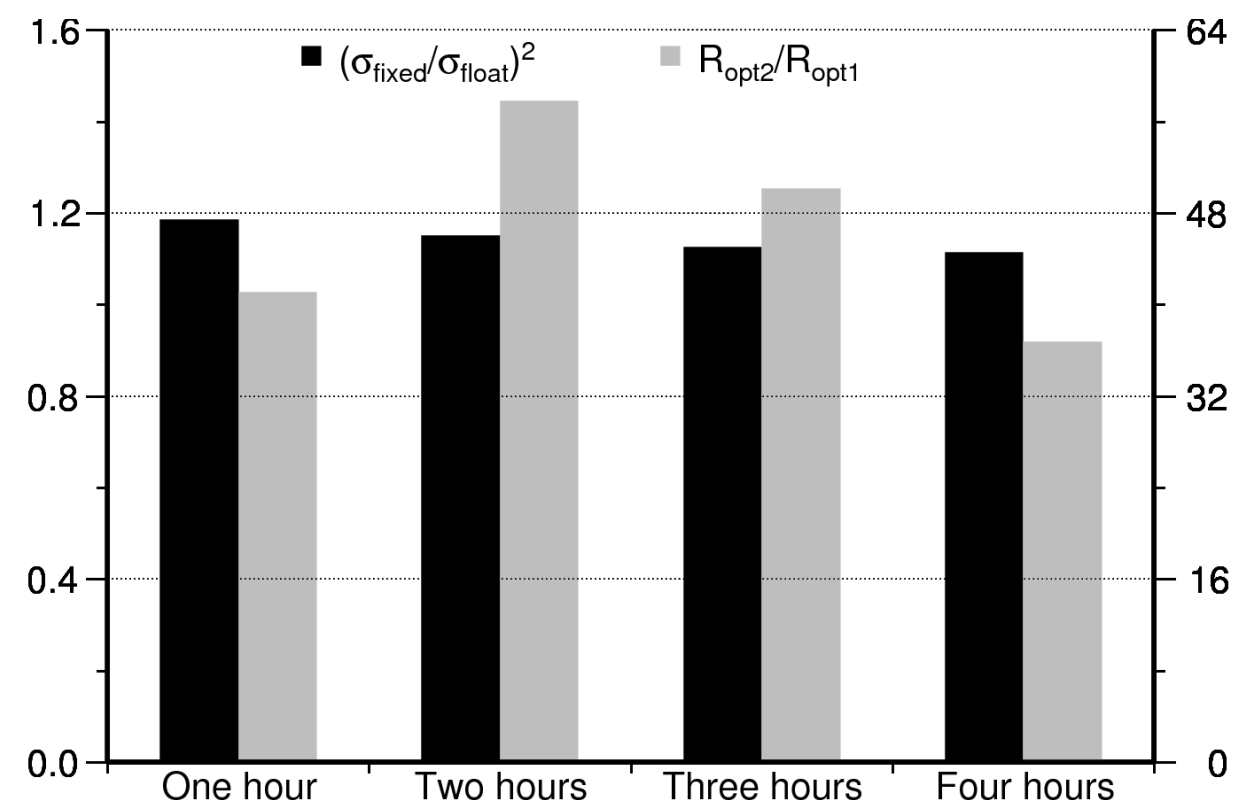

Fig. 2. Mean values of the test statistics for ambiguity validation in all fixed solutions when different short observation periods are used. Note that the black bars refer to the left axis while the grey bars refer to the right axis. 


\section{Positioning accuracy}

Table 3 shows the mean positioning accuracy of all PPP solutions at all test stations for the different short observation periods. As was demonstrated by [12], the positioning accuracy can be significantly improved through ambiguity resolution in hourly PPP. Table 3 further shows that, even in 4-hourly PPP, the accuracy of the East component is still improved significantly by approximately $66.7 \%$ and the $3 \mathrm{D}$ improvement achieves $35.7 \%$. These results confirm that the positioning accuracy, especially for the horizontal components, benefits significantly from the ambiguity resolution in the short-period static PPP.

In addition, it is clear in Table 3 that increasing the observation period significantly improves the positioning accuracy in the float solutions. When the observation period increases from one hour to two, three and four hours, the 3D positioning accuracy is improved markedly from $5.0 \mathrm{~cm}$ to $2.9 \mathrm{~cm}, 1.9 \mathrm{~cm}$ and $1.4 \mathrm{~cm}$, respectively. However, this pattern is not evident in the fixed solutions. Especially, the accuracy of each horizontal component remains around $4 \mathrm{~mm}$ for all short observation periods, and only the vertical component exhibits the trend of accuracy improvement with the increased observation period. Therefore, it is demonstrated that, for the fixed solutions, the horizontal positioning accuracy is hardly influenced by the length of the observation periods when they are over one hour, while the vertical one is improved when the observation periods are increased from one to four hours.

Table 3. Mean positioning accuracy for different short observation periods

\begin{tabular}{lcccccccc}
\hline \multirow{2}{*}{ Observation period } & \multicolumn{3}{c}{ Float solution (cm) } & \multicolumn{4}{c}{ Fixed solution $(\mathrm{cm})$} \\
& East & North & Up & 3D & East & North & Up & 3D \\
\hline One hour & 3.8 & 1.5 & 2.8 & 5.0 & 0.5 & 0.5 & 1.4 & 1.6 \\
Two hours & 2.0 & 0.9 & 1.8 & 2.9 & 0.4 & 0.4 & 1.0 & 1.2 \\
Three hours & 1.3 & 0.6 & 1.3 & 1.9 & 0.3 & 0.4 & 0.9 & 1.0 \\
Four hours & 0.9 & 0.5 & 1.0 & 1.4 & 0.3 & 0.4 & 0.8 & 0.9 \\
\hline
\end{tabular}

\section{Degraded solutions}

[12] showed that correct ambiguity resolution may lead to degraded, rather than improved, positioning accuracy in the fixed solutions compared with the float ones. In this study, these solutions are defined as degraded solutions and are identified when the $3 \mathrm{D}$ positioning accuracy degradation exceeds $1.0 \mathrm{~cm}$. A degradation of less than $1.0 \mathrm{~cm}$ is considered minimal and thus excluded from the statistics related to the degraded solutions.

Table 4 shows the number of degraded solutions, the percentages in all solutions with successful ambiguity resolution, and the mean and maximum accuracy degradation for different short observation periods. In the hourly solutions, the percentage of the degraded solutions is up to $1.1 \%$ and the maximum accuracy degradation is up to about $3 \mathrm{~cm}$. When the observation period is increased to three hours, however, the percentage is decreased to $0.7 \%$ and the maximum accuracy degradation is reduced to $1.7 \mathrm{~cm}$. As is expected, the least percentage of $0.2 \%$ corresponds to the observation period of four hours. As was discussed in [12], degraded solutions are closely related to the estimation of zenith tropospheric delays. It is well-known that a long observation period is beneficial to the highly accurate estimates of the zenith tropospheric delays. Hence, the occurrence of degraded solutions can be decreased through prolonging the observation period, rather than fixing the zenith tropospheric delays to precisely known values as was discussed in $[12]$. 
Table 4. Number of degraded solutions, the percentages in all solutions with successful ambiguity resolution, mean accuracy degradation and maximum accuracy degradation for different short observation periods

\begin{tabular}{lllll}
\hline Observation period & Number & Percentage & Mean $(\mathrm{cm})$ & Max. $(\mathrm{cm})$ \\
\hline One hour & 22 & $1.1 \%$ & 1.5 & 2.9 \\
Two hours & 11 & $1.1 \%$ & 1.5 & 2.7 \\
Three hours & 5 & $0.7 \%$ & 1.3 & 1.7 \\
Four hours & 1 & $0.2 \%$ & 1.2 & 1.2 \\
\hline
\end{tabular}

Moreover, Table 5 shows the percentages of the solutions with accuracy degradation in the East, North or Up directions and the corresponding mean degradation in each direction. It can be found that all degraded solutions suffer accuracy degradation for the Up component which also exhibits the largest degradation among the three components. Thus it is demonstrated that the accuracy degradation mainly and markedly occurred in the Up component, which explains why the degraded solutions are closely associated with the zenith tropospheric delay estimation that is strongly correlated with the Up component in a least squares adjustment.

Table 5. Percentages of the solutions with accuracy degradation in the East, North or Up directions and the corresponding mean degradation in each direction

\begin{tabular}{lcccccc}
\hline \multirow{2}{*}{ Observation period } & \multicolumn{3}{c}{ Percentage } & \multicolumn{3}{c}{ Mean degradation (cm) } \\
& East & North & Up & East & North & Up \\
\hline One hour & $45.5 \%$ & $59.1 \%$ & $100 \%$ & 0.4 & 0.5 & 1.8 \\
Two hours & $45.5 \%$ & $54.5 \%$ & $100 \%$ & 0.5 & 0.6 & 1.6 \\
Three hours & $40.0 \%$ & 0 & $100 \%$ & 0.7 & 0 & 1.9 \\
Four hours & 0 & 0 & $100 \%$ & 0 & 0 & 1.8 \\
\hline
\end{tabular}

\section{CONCLUSIONS}

Although daily static PPP is capable of providing millimetre positioning accuracy, PPP using short periods of observations, such as one to four hours, can hardly achieve such high accuracy when the real-valued ambiguity estimates are kept in the final solutions. However, if the non-integer uncalibrated phase delays are separated from the real-valued ambiguity estimates, integer ambiguity resolution becomes possible, thus leading to improved positioning accuracy.

This study applies the ambiguity resolution strategy to a single station in order to compare the performance of the ambiguity-fixed static PPP with different short observation periods within a regional network of base stations.

It is demonstrated that fairly reliable ambiguity resolution can be achieved when the observation period is over one hour. When the observation period is over three hours, the success rate of PPP ambiguity resolution can even reach $100.0 \%$.

In the fixed solutions of short-period static PPP, over one hour of data are sufficient for the horizontal position components to achieve an accuracy of better than $1.0 \mathrm{~cm}$ while over three hours of data are still required for the vertical component to achieve such accuracy. Moreover, increasing the observation periods can hardly improve the horizontal accuracy, but clearly improves the vertical accuracy in the fixed solutions.

In addition, for degraded solutions, the accuracy degradation mainly and markedly occurred in the Up component. Increasing the observation periods to three hours can reduce the occurrence of these degraded solutions to less than $1.0 \%$ of all solutions with successful ambiguity resolution. 
Therefore, it is proposed that, in engineering applications when the ambiguity resolution at a single station is implemented, three hours of observations for static PPP should be used if a reliable millimetre positioning accuracy is required.

\section{ACKNOWLEDGEMENTS}

This research is supported by a scholarship provided to Mr. Geng by the University of Nottingham. The authors also would like to thank EUREF and IGS communities for the provision of GPS data and products.

\section{References}

1. Blewitt, G., 1989. Carrier phase ambiguity resolution for the global positioning system applied to geodetic baselines up to $2000 \mathrm{~km}$. Journal of Geophysical Research. 94(B8):10187-10203

2. Bruyninx, C., Becker, M., Stangl, G., 2001. Regional densification of the IGS in Europe using the EUREF permanent GPS network (EPN). Physics and Chemistry of the Earth Part A. 26(68):531-538

3. Collins, P., 2008. Isolating and estimating undifferenced GPS integer ambiguities. Proceedings of ION National Technical Meeting, 28-30 September, San Diego, CA.

4. Collins, P., Lahaye, F., Héroux, P. and Bisnath, S., 2008. Precise point positioning with ambiguity resolution using the decoupled clock model. Proceedings of ION GNSS 2008, 16-19 September, Savannah, GE.

5. Creager, G.J. and Maggio, R.C., 1998. How long must we wait? Duration of GPS observations for long GPS baselines. Proceedings of $54^{\text {th }}$ Annual Navigation Meeting. Denver, USA, pp.467-475.

6. Dong, D. and Bock, Y., 1989. Global positioning system network analysis with phase ambiguity resolution applied to crustal deformation studies in California. Journal of Geophysical Research. 94(B4):3949-3966.

7. Eckl, M.C., Snay, R.A., Soler, T., Cline, M.W. and Mader, G.L., 2001. Accuracy of GPSderived relative positions as a function of interstation distance and observing-session duration. Journal of Geodesy, 75(12):633-640.

8. Gabor, M.J. and Nerem, R.S., 1999. GPS carrier phase ambiguity resolution using satellitesatellite single difference. Proceedings of ION GPS 1999, 14-17 September, Nashville, TN.

9. Ge, M., Gendt, G., Rothacher, M., Shi, C. and Liu, J., 2007. Resolution of GPS carrier-phase ambiguities in precise point positioning (PPP) with daily observations. Journal of Geodesy. 82(7):389-399.

10. Geng, J., Shi, C., Zhao, Q. and Liu, J., 2006. Integrated adjustment of LEO and GPS in precision orbit determination. Proceedings of VI Hotine-Marussi Symposium on Theoretical and Computational Geodesy, Springer-Verlag, Berlin, Heidelberg.

11. Geng, J., Shi, C., 2008. Performance of hourly precise point positioning with ambiguity resolution. Proceedings of ION GNSS 2008, 16-19 September, GE.

12. Geng, J., Teferle, F.N., Shi, C., Meng, X., Dodson, A.H. and Liu, J., 2009. Ambiguity resolution in precise point positioning with hourly data. GPS solutions. Doi:10.1007/s10291009-0119-2.

13. Ghoddousi-Fard, R. and Dare, P., 2006. Online GPS processing services: an initial study. GPS solutions, 10(1):12-20.

14. Han, S., 1997. Quality-control issues relating to instantaneous ambiguity resolution for realtime GPS kinematic positioning. Journal of Geodesy. 71(6):351-361.

15. Kouba, J. and Héroux, P., 2001. Precise point positioning using IGS orbit and clock products. GPS solutions, 5(2):12-28.

16. Laurichesse, D. and Mercier, F., 2007. Integer ambiguity resolution on undifferenced GPS phase measurements and its application to PPP. Proceedings of ION GNSS 2007, 25-28 September, Fort Worth, TX.

17. Lazio, P., 2007. Constraining network adjustments to OPUS-RS coordinate observations. Journal of Surveying Engineering, 133(3):106-113.

18. McCarthy, D.D. and Petit, G., 2004. IERS conventions 2003. IERS technical note No. 32. Frankfurt am Main: Verlag des Bundes für Kartographie und Geodäsie. 127 pages. 
19. Melbourne, W.G., 1985. The case for ranging in GPS-based geodetic systems. Proceedings of First International Symposium on Precise Positioning with the Global Positioning System, 1519 April, Rockville.

20. Niell, A.E., 1996. Global mapping functions for the atmospheric delay at radio wavelengths. Journal of Geophysical Research, 101(B2):3227-3246.

21. Soler, T., Michalak, P., Weston, N.D., Snay, R.A. and Foote, R.H., 2006. Accuracy of OPUS solutions for 1- to 4-h observing sessions. GPS solutions, 10(1):45-55.

22. Teferle, F.N., Orliac, E.J., Bingley, R.M., 2007. An assessment of Bernese GPS software precise point positioning using IGS final products for global site velocities. GPS solutions. 11(3):205-213

23. Tétreault, P., Kouba, J., Héroux, P. and Legree, P., 2005. CSRS-PPP: an internet service for GPS user access to the Canadian Spatial Reference Frame. Geomatica, 59(1):17-28.

24. Teunissen, P.J.G., 1994. A new method for fast carrier phase ambiguity estimation. Proceedings of IEEE Position, Location and Navigation Symposium, 11-15 April, pp:562-573.

25. Teunissen, P.J.G. and Kleusberg, A., 1997. GPS for Geodesy (2 ${ }^{\text {nd }}$ Edition). Springer-Verlag, Berlin, Heidelberg, New York. 650 pages.

26. Verhagen, S., 2004. Integer ambiguity validation: an open problem? GPS solutions, 8(1):36-43.

27. Wübbena, G., 1985. Software development for geodetic positioning with GPS using TI-4100 code and carrier measurements. Proceedings of First International Symposium on Precise Positioning with the Global Positioning System, 15-19 April, Rockville.

28. Zumberge, J.F., Heflin, M.B., Jefferson, D.C., Watkins, M.M. and Webb, F.H., 1997. Precise point positioning for the efficient and robust analysis of GPS data from large networks. Journal of Geophysical Research, 102(B3):5005-5017. 